\title{
VISIONES DEL USO DE LAS TIC PARA LA EDUCACIÓN INCLUSIVA DESDE LA PERSPECTIVA DOCENTE: EL CASO DE GRECIA
}

\section{VISIONS OF THE USE OF ICT FOR INCLUSIVE EDUCATION: THE CASE OF GREECE VISÕES DO USO DE TIC PARA A EDUCAÇÃO INCLUSIVA: O CASO DA GRÉCIA}

\author{
Verónica Marín Díaz \\ Universidad de Córdoba, Espanha \\ vmarin@uco.es \\ Evangelia Vagena \\ Secondary Education Directorate of Pella, Grécia \\ eva.vagena@gmail.com \\ Sebastían Rubio García \\ Universidad de Córdoba, Espanha \\ sjrubio@uco.es
}

RESUMEN: La educción inclusiva hoy pasa unilateralmente por imbricar su práctica con las Tecnologías de la Información y la Comunicación. Sin embargo, para que esto se pueda producir las percepciones que los docentes tienen de las tecnologías van a marcar su empleo en la dinámica educativa. El objetivo principal de esta investigación es recopilar la opinión que tienen los profesores de Educación Secundaria de la localidad Atenas y Tesalónica (Grecia) sobre el empleo de las TIC en ambientes educativos inclusivos. A través de un diseño ex post facto y mediante un estudio descriptivo, inferencial y correlacional, se ha administrado un cuestionario creado ad hoc a una muestra de 377 docentes de Enseñanza Secundaria de Grecia, seleccionados a través de un muestreo de tipo incidental. El principal resultado alcanzado indica la no existencia de diferencias en sus visiones en función de la edad o del género, y que consideran que sus estudiantes están mejor formados digitalmente que ellos. Se puede concluir que, al igual que sucede en otros contextos los docentes helénicos demandan una formación clave para la utilización de las TIC en los entornos inclusivos en los que trabajan.

PALABRAS CLAVE: TIC. Aprendizaje. Educación inclusiva. Formación del profesorado.

\begin{abstract}
Inclusive education today unilaterally goes through overlapping its practice with Information and Communication Technologies. However, for this to occur, teachers' perceptions of technologies will herald their use in educational dynamics. The main objective of this research is to get the opinion that the teachers of Secondary Education of the locality Athens and Thessalonica (Greece) have about the use of ICT in inclusive educational environments. Through an ex post facto design and through a descriptive, inferential and correlational study, a questionnaire created ad hoc has been administered to a sample of 377 secondary school teachers from Greece, selected through incidental sampling. The main result achieved indicates the non-existence of differences in their visions based on age or gender, and that they consider that their students are better digitally trained than they are. It can be concluded that, as in other contexts, Hellenic teachers demand key training for the use of ICT in the inclusive environments in which
\end{abstract}


they work.

KEYWORDS: ICT. Training. Inclusive education. Teacher training.

RESUMO: A educação inclusiva hoje passa de forma unilateral pela sobreposição de sua prática com as Tecnologias de Informação e Comunicação. No entanto, para que isso ocorra, as percepções que os professores têm das tecnologias vão marcar a sua utilização na dinâmica educacional. O objetivo principal desta pesquisa é compilar a opinião de professores do ensino médio de Atenas e Salonica (Grécia) sobre o uso das TIC em ambientes educacionais inclusivos. Por meio de um desenho ex post facto e por meio de um estudo descritivo, inferencial e correlacional, um questionário elaborado ad hoc foi aplicado a uma amostra de 377 professores do ensino médio da Grécia, selecionados por amostragem incidental. O principal resultado alcançado indica que não há diferenças nas suas visões com base na idade ou no sexo, e que consideram que os seus alunos têm melhor formação digital do que eles. Pode-se concluir que, como em outros contextos, os professores helênicos exigem uma formação fundamental para o uso das TIC nos ambientes inclusivos em que trabalham.

PALAVRAS-CHAVE: TIC. Aprendizagem. Educação inclusiva. Formação de professores.

\section{Introducción}

La educción inclusiva del siglo XXI debe partir de la búsqueda de respuestas, a los interrogantes que la diversidad de las aulas en particular y de los centros educativos, en general, presentan. Las diferentes declaraciones que a lo largo de los últimos veinte años se han ido generando tienen todas el mismo fin, proveer de una educación a todos por igual y acercar los procesos de enseñanza a la heterogeneidad del alumnado, todo ello desde una perspectiva de colaboración, pluralidad y fomento de la máxima participación de todos los implicados en los procesos formativos. Como señala Echeita (2017, p. 17) es una perspectiva que nos debe permitir analizar los desafíos que la educación hoy nos lanza. Y en pro de dar respuesta a dicho desafíos las Tecnologías de la Información y la Comunicación (en adelante TIC) se perfilan como un recurso que ayudará no solo a ello, sino también en la consecución de "un contexto que reconoce la creación individual y la colectiva, que estimula tanto fuera como dentro del aula" (GARCíA, 2019, p. 78) y que busca la mejor formación de, por y para todos.

En este escenario la figura del docente cobra relevancia, dado que sus visiones, experiencias, creencias y perspectivas van a determinar el empleo de las herramientas tecnológicas. En consecuencia, es necesario analizar el escenario en que la educación inclusiva digital se debe o puede desarrollarse. Por otra parte, no en todos los países se está llevando a cabo o poniendo en marcha el proceso inclusivo digital de la misma forma. En el caso de la Unión Europea encontramos diferentes perspectivas no solo del propio concepto de educación inclusiva, sino también del tratamiento que de ella se debe dar, así como de los aspectos a priorizar. De este modo, encontramos en general que la formación del profesorado en educación inclusiva vinculada al uso y consumo de recursos digitales pasa por la voluntariedad del docente en promover su capacitación, aspecto este común a todos los integrantes de la unión. Los diferentes países ofrecen una formación básica bajo diversas denominaciones, pero todas conducentes a la obtención de un título superior o 
medio, pero no una especialización, esta depende de los diseños curriculares que las universidades hayan planeado. Así, podemos encontrar en España, por ejemplo, que la especialización en el ámbito de la educación inclusiva se centra en el último año del título de Grado de Maestro de Educación Primaria, mientras que en Grecia existen titulaciones específicas para ello, así como diplomas de título medio. Como vemos la diversificación curricular así como el planteamiento formativo, finalmente recae en la figura del docente.

En consecuencia, estudiar y/o analizar la visión que el profesorado tiene de las TIC en general y dentro del ámbito de la inclusión en particular se vuelve aspecto capital si los estados quieren desarrollar políticas sociales y educativas públicas, que se acerquen a la realidad inclusiva.

Es por ello, que desde este artículo se presenta un estudio realizado entre profesores griegos con el objeto de determinar la visión que tienen de la utilización de las TIC en ambientes educativos tanto de carácter general como inclusivos, y en este aspecto más concretamente en el ámbito de las discapacidades intelectuales y cognitivas.

\section{Preliminares: el sistema educativo griego}

Al igual que el sistema educativo español o el francés, el helénico consta de 4 etapas educativas diferenciadas, siendo la Educación Primaria y la Secundaria de carácter obligatorias y comprendiendo la horquilla de edad de los 6 a los 15 años. Igualmente, es un sistema de capacitación gratuito, organizado en enseñanza pública y privada. Los estudiantes que aprueban el nivel primario pasan directamente al primer curso de la secundaria, al igual que sucede en España, por citar otro país comunitario.

En el caso de los docentes sus procesos de formación estriban en una titulación de grado - desde que se puso en marcha el Espacio Europeo de Educación Superior -, capacitación que puede verse complementada haciendo bien una segunda titulación, un máster y posteriormente el doctorado. Con respecto al acceso al ejercicio docente, este puede realizarse por tres vías, la contratación directa bien a tiempo parcial bien a tiempo completo en las instituciones de carácter privado, a través del acceso a la función pública o como autónomos contratados de manera externa.

El sistema educativo griego parte de una organización centralizada en el gobierno del país, quien ostenta todas las competencias en esta materia. Esta circunstancia genera grandes dificultades a la hora de desarrollar planes formativos en general e inclusivos en particular (KORKOUTAS, 2011), dado que se necesita una autorización expresa por parte del gobierno para poder llevar a cabo diversas iniciativas académicas que impliquen a alumnos menores de edad. No obstante, las acciones de mejora se van plasmando, un ejemplo de esos cambios es el haber otorgado a los directores de los centros educativos más competencias en materia de gestión y de formación, de cara a poder resolver las nuevas situaciones que el desarrollo de la sociedad presenta y demanda. Igualmente en el ámbito de la educación inclusiva se están implementando diferentes modelos de actuación, siendo el denominado "instrucción paralela" o "enseñanza paralela" el que más se utiliza en el país (PADELIADU; PAPANIKOLAOU; GIAZITZIDOU, 2014), además de la creación de Centros de Apoyo Educativo y de Orientación, los Centros Regionales de Planificación Educativa y el Coordinador del Proyecto Educativo como responsables de llevar a cabo la promoción de la educación inclusiva en todo el país (PAPAGEORGIOU, 
2019).

Grecia, a lo largo de esta última década, ha ido desarrollando diversas normativas de cara a la protección de los menores y de la asunción de la educación inclusiva como un punto en su agenda educativa. Así, por ejemplo, la Ley 4488/2017 hace hincapié en la no vulneración de los derechos de las personas con discapacidad y asegurar su participación plena en la sociedad.

Sin embargo, si bien a día de hoy estas lagunas o hándicap se van solventando, la falta de una formación continua de calidad es algo que sigue siendo un hecho y una demanda por los profesionales de la educación en Grecia (SALTA; KOULOUGLIOTIS, 2014).

Por otra parte, si nos detenemos en la vinculación de la enseñanza en general con las TIC, el gobierno griego desde 2008 ha ido promoviendo diversas acciones de formación para los docentes; no obstante, al igual que pasa en otros países, estos quedan a la voluntariedad de los docentes (SALTA; KOULOUGLIOTIS, 2014).

Poniendo el acento en el binomio educación inclusiva+TIC la visión que se tiene a nivel general en Europa y en Grecia en particular, es que es escasa (FIGUEREDO, 2018). El trabajo llevado a cabo por Figueredo (2018) señala que este país en concreto, ha centrado sus esfuerzos en la creación de redes de profesores que promuevan la educación inclusiva, pero no ponen el acento en la combinación de ambos elementos.

\section{La inclusión digital educativa}

La UNESCO en 2017 llamaba la atención sobre los dos principios fundamentales que debían vertebrar las políticas, planes y prácticas educativas, de un lado la educación inclusiva y de otro la equidad. Centrando nuestra atención en el primero de ellos, comprobamos que este se encuentra vinculado al desarrollo que la sociedad tiene en todas sus esferas, siendo la tecnológica la más emblemática, si cabe. Las Tecnologías de la Información y la Comunicación (en adelante TIC) contribuyen a igualar y promover la participación en la sociedad en todos los niveles (vida social, empleo, participación política, salud, etc.) (ÁVALOS et al., 2018).

El logro de una sociedad inclusiva digital pasa por promover políticas educativas, económicas y sociales que generen que aspectos como la brecha digital se eliminen o en su defecto se vaya diluyendo. Compartimos con Thomas, Barraket, Ewing, MacDonald, Mundell y Tucker (2016), que ello es un elemento complejo y multifacético, pero esto no quiere decir que sea imposible de alcanzar. El reto de lograrlo pasa por una formación en el uso de los recursos digitales, lo que en palabras de Fuente-Cobo (2017), sería promover el empoderamiento digital de la sociedad y este pasaría, necesariamente, por potenciar una educación que aproveche las ventajas que las TIC en general y sus recursos digitales en particular ofrecen a toda la población.

Centrando nuestra atención en la esfera educativa debemos ser conscientes de que, como sostenía Fernández en 2013 , hay que promover una enseñanza de calidad, lo que implica la movilización de recursos tanto sociales como económicos, políticos y educativos, de modo que cubran y den respuesta a las demandas que la e-sociedad, en general, plantea al sistema educativo. Y ello pasa por movilizar las TIC en las acciones formativas que los profesionales de la educación desarrollan, independientemente del 
nivel educativo en que nos ubiquemos.

Por otra parte, hablar de educación inclusiva digital supone en palabras de Zappata, Köppel y Suchodoliski (2011, p. 20) hacerlo de "un proceso de apropiación de herramientas y conocimientos, y la construcción de concepciones tendentes a incorporar los recursos materiales y digitales como contenidos flexibles, adaptables y transversales". En él los avances sociales, las posibilidades de elección, la disponibilidad de recursos, la formación, el interés, el atractivo de la interfaz, entre otros aspectos, implicarán su incorporación a ámbitos inclusivos (MARÍN-DÍAZ, 2019).

En este sentido hay que manifestar hoy que el binomio educación inclusiva+TIC es una realidad incuestionable (MARÍN, 2018), prueba de ello es el número de investigaciones y acciones de innovación educativa que se están llevando a cabo (CABERO; VALENCIA, 2019; DA SILVA; DE CARVALHO; RAMOS, 2020; FERNÁNDEZ BATANERO; REYES REBOLLO; EL HOMRANI, 2018; HAMBURG; BUCKSCH, 2017; MARÍN-DÍAZ, 2017; CÁMARA; DÍAZ; ORTEGA, 2017; MARTÍNEZ; GUTIÉRREZ; FERNÁNDEZ, 2018; PASSEY, 2017; RICO; BOSAGAIN, 2018; ROMERO; GARCÍA, GONZÁLEZ; LOZANO, 2018). En general, desde todas ellas se pone de manifiesto la necesidad de emplear los recursos digitales en la educación para buscar las fórmulas de cara a que los maestros/profesores en formación las incorporen en sus propios procesos de aprendizaje y durante su futuro ejercicio profesional, y para que los docentes en ejercicio las imbriquen en sus metodologías. También, se deben utilizar para reclamar actuaciones y mejoras a los teóricos y políticos de la educación en pro de una inclusión digital plena.

Es interesante indicar que entre las principales ventajas del empleo de las TIC en la educación inclusiva, giran en torno a la mejora del auto-concepto que logra el alumno de sí mismo, a un progreso positivo de su rendimiento académico, a permitir la adaptación del currículo a la especificidad del estudiante, facilitar la individualización de la enseñanza, favorecen los principios de accesibilidad, a potenciar la autonomía del alumno, etc. (TOLEDO, 2013; PEGALAJAR, 2015; SAMPEDRO; MALDONADO, 2017). Estos aspectos hacen que los sistemas educativos avancen, al igual que las metodologías docentes, pues el fin último es que los contenidos sean comprensibles y útiles por cualquier sujeto, ya que no debemos perder de nuestro ángulo de visión que las TIC son medidoras del proceso de aprendizaje y que se encuentran vinculadas a las capacidades de los docentes y de los estudiantes (CUMMING TONES; DAY; HECK, 2018). Pues como señalaba hace más de una década Parrilla (2002) la inclusión es un reto, que no es otro que el de crear un espacio donde converjan múltiples iniciativas y disciplinas, y hoy ello pasa por las TIC y por la inquietud del docente de incorporarlas. Somos diferentes y diversos en nuestra visa social y como no podía ser de otro modo, también en nuestra forma de aprender, y eso es lo que enriquece a las sociedades. Leiva y Gómez (2015, p. 195) señalan que "la meta o misión de la educación debe centrarse en mejorar la calidad de vida de cada alumno... interesa el aprendizaje para el óptimo desarrollo personal, la felicidad y el bienestar integral de individuos y colectivos", y ello pasa por incorporar las TIC al aula.

\section{Método}

La presente investigación tiene como principal objetivo recopilar la opinión de los 
profesores de Educación Primaria y Secundaria de la localidad Atenas y Tesalónica (Grecia) tienen sobre el empleo de las TIC en ambientes educativos inclusivos.

Derivado de este objetivo nos hemos planteado las siguientes hipótesis de trabajo en modo de interrogante: inclusivas?

¿El género determina el posicionamiento ante el uso de las TIC en las aulas

¿La edad puede determinar el empleo de las TIC en las metodologías docentes desarrolladas en entornos inclusivos?

¿Puede la experiencia profesional (años de trabajo) determinar la visión que el docente tiene de la incorporación de las TIC a su dinámica de trabajo en las aulas inclusivas?

La naturaleza de esta investigación es de corte cuantitativo, la cual se acoge a un método de estudio ex post facto, de esta forma la consecución del objetivo se alcanzará a posteriori como señala Mateo (2012).

Con respecto a la población objeto de estudio son profesores de los niveles de educación Secundaria de las ciudades de Atenas y Tesalónica. La muestra ha sido extraída empleando un método incidental (MANTILLA, 2015), de los cuales han participado 377 docentes, y de estos el $84.1 \%$ son mujeres y el $15.9 \%$ son hombres (M.=.84, D.T.=3.66). A la vez el 33.4\% tienen entre 31-35 años, el 22.8\% 36-40 años, el $14.3 \%$ se ubica entre los $46-50$ años, el 10.1 tiene más de 51 años de edad y el $6.4 \%$ menos de 30 (M.=3.26, D.T.=1.462). Con respecto a los estudios que poseen los profesores, y teniendo en cuenta el marco formativo griego, los mayores perfiles profesionales lo encontramos en el ámbito de la Filología general con un $20.4 \%$ y un 17.2\% (M.=.84, D.T.=3.66), que tiene la especialidad de profesor de educación especial (ver Tabla 1).

Tabla 1: Distribución de la muestra en función de sus estudios.

\begin{tabular}{|l|c|c|}
\hline & F. & $\%$ \\
\hline Filología Inglesa & 5 & 1,3 \\
\hline Biología & 3 & .8 \\
\hline Filología Francesa & 2 & .5 \\
\hline Filología Alemana & 5 & 1.3 \\
\hline Geología & 4 & 1.1 \\
\hline Agricultura & 5 & 1.3 \\
\hline Profesor de educación especial & 65 & 17.2 \\
\hline Profesor de Arte & 6 & 1.6 \\
\hline Profesor de Religión & 1 & .3 \\
\hline Profesor de Nutrición & 2 & .5 \\
\hline Profesor de producción animal & 3 & .8 \\
\hline Profesor de Peluquería & 1 & .3 \\
\hline Profesor de Educación & 1 & .3 \\
\hline Profesor de cursos de Armanda & 3 & .8 \\
\hline Profesor de Economía & 2 & .5 \\
\hline Profesor de Psicología Educativa & 22 & 5.8 \\
\hline Educación Física & 26 & 6.9 \\
\hline
\end{tabular}


Belo Horizonte, v. 13, n. 3, p. 181-199, set.-dez. 2020 - ISSN 1983-3652 DOI : $10.35699 / 1983-3652.2020 .25117$

\begin{tabular}{|l|c|c|}
\hline Ecología & 3 & .8 \\
\hline Teología & 7 & 1.9 \\
\hline Trabajo social & 6 & 1.6 \\
\hline Sociología & 2 & .5 \\
\hline Matemáticas & 46 & 12.2 \\
\hline Música & 6 & 1.6 \\
\hline Enfermería & 12 & 3.2 \\
\hline Economía & 7 & 1.9 \\
\hline Informática & 48 & 12.7 \\
\hline Ingeniería civil & 3 & .8 \\
\hline Filología & 77 & 20.4 \\
\hline Química & 4 & 1.1 \\
\hline
\end{tabular}

Fuente: Elaboración propia.

En la línea de la formación que poseen los participantes encontramos que el $62.1 \%$ tiene estudios de máster frente al 3.7\% que solo tiene los estudios superiores antes indicados ( $\mathrm{M}=2.44 ; \mathrm{D} . \mathrm{T} .=1.285)$.

Con respecto a la formación vinculada con las TIC el 73.5\% señaló haber asistido a actividades formativas en el último año o semestre, frente al 3.4\% que se ha ido formando de manera autodidacta y el $3.7 \%$ que no ha recibido formación alguna en el uso de las TIC (M= 3.01; D.T.=1.025).

La vinculación de los docentes con las instituciones educativas en Grecia, son bien a tiempo completo bien a parcial, tanto con los centros públicos como privados. Atendiendo a este dato, el $65 \%$ de la muestra participante es docente a tiempo completo en un centro privado y a tiempo parcial el $5.6 \%$, mientras que en los centros públicos encontramos el $24.1 \%$ de los participantes. También se contempla el trabajo como autónomo para centros públicos o privados, siendo el $3.7 \%$ de los profesores los que trabajan bajo esa modalidad ( $\mathrm{M}=1.91$; D.T.=1.418).

Atendiendo a los años de experiencia en el ámbito educativo (ver Gráfico 1), en general vemos que la muestra se ubica en la horquilla de 6 a 10 años de experiencia profesional, siendo el 72.4\%, mientras que 16.5\% lleva entre 1 año y 5 trabajando en el ámbito educativo ( $\mathrm{M}=1.98$; D.T.=.676). En lo que se refiere a su vinculación al ámbito de la educación inclusiva, vemos que sucede igual que con los datos generales, la mayor parte de la muestra se ubica entre 6 a 10 años de experiencia en esa área (53.8\%) y 1-5 años (44\%), frente al .6\% que se ubica en el intervalo de 16 a 25 años de trabajo $(M=1.59$; D.T.=.568). 
Gráfico 1: Distribución de la muestra según la edad.

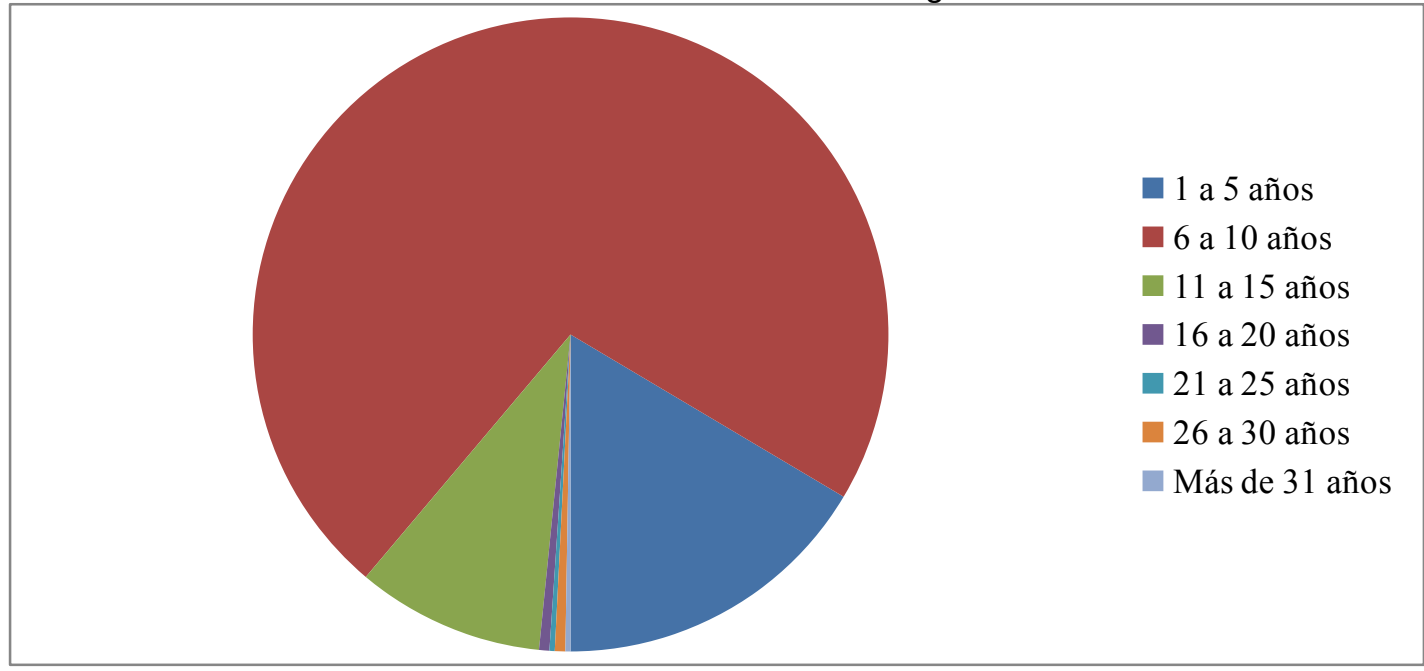

Fuente: Elaboración propia.

El instrumento para la recogida de los datos fue un cuestionario creado ad hoc por los investigadores. Este se conformó por 40 ítems, de los cuales los 8 primeros correspondían a variables socio-demográficas y descriptivas del entorno educativo del centro y el resto se distribuye en 4 dimensiones. La escala de respuesta que se ha empleado ha sido tipo Likert de 5 opciones, donde 1 correspondía a totalmente en desacuerdo y 5 a totalmente de acuerdo.

La fiabilidad y la validez del instrumento diseñado para este estudio se ha realizado a través de diferentes procesos y análisis ejecutados. La fiabilidad se ha obtenido a través de la prueba Alpha de Cronbach, la cual ha arrojado un peso de .821, mostrando un alto valor (MATEO, 2012).

Con respecto a la validez de constructo se ha realizado un Análisis Factorial Exploratorio (AFE) con un método de extracción de máxima verosimilitud y rotación varimax. siguiendo las pautas de Hernández, Fernández y Baptista (2006), el cálculo previo del índice de Kaiser- Meyer y Olkin (KMO =0.869), la prueba de esfericidad de Bartlett $(p<.001)$ denotan significatividad; estas cual han arrojado un total de 4 factores que explican un $60.523 \%$ de la varianza total explicada (ver Tabla 2).

Tabla 2: Distribución ítem por factores.

\begin{tabular}{|c|c|c|c|c|}
\hline \multirow{2}{*}{ Ítem } & \multicolumn{4}{|c|}{ Factores } \\
\hline & 1 & 2 & 3 & 4 \\
\hline $\begin{array}{l}\text { 1. Soy cauteloso sobre el uso de las TIC en la educación, porque creo que } \\
\text { las limitan las interacciones sociales y mejoran el aislamiento }\end{array}$ & .479 & & & \\
\hline Evito usar las TIC porque no tengo el conocimiento necesario & 476 & & & \\
\hline $\begin{array}{l}\text { 3. Si tuviera una computadora en mi clase, la usaría en el proceso de } \\
\text { enseñanza }\end{array}$ & .460 & & & \\
\hline $\begin{array}{l}\text { 4. Las TIC son útiles en los programas de educación inclusiva mejorando la } \\
\text { calidad de la enseñanza }\end{array}$ & 455 & & & \\
\hline El uso de las TIC en el aula de hoy incluye actividades inclusivas & .454 & & & \\
\hline $\begin{array}{l}\text { 6. El uso de nuevas herramientas digitales en programas de enseñanza } \\
\text { inclusiva me interesa }\end{array}$ & 451 & & & \\
\hline 7. El uso de las TIC en el proceso de enseñanza en general me interesa & .448 & & & \\
\hline
\end{tabular}


8. No me gusta usar las TIC durante la enseñanza

9. Necesito probar las nuevas herramientas digitales antes de aplicarlas en la práctica docente

10. Las computadoras ayudan a los estudiantes con diferentes estilos de aprendizaje a aprender mejor y participar más

11. No usaré las TIC en la enseñanza, porque me siento inseguro sobre su aplicación en la educación inclusiva

12. La inclusión suena bien en teoría, pero no funciona bien en la práctica

13. Los estudiantes con discapacidades deben ser educados en clase regular

14. Los estudiantes con discapacidad pueden obtener una mejora académica debido a la educación inclusiva y a la implementación de las TIC en el aula

15. El uso frecuente de las TIC contribuye a la socialización de los estudiantes con discapacidad

16. La implementación de las TIC puede mejorar el nivel de aprendizaje de los estudiantes con discapacidades

17. Los escenarios de enseñanza con TIC atraen el interés de los estudiantes con discapacidad

18. Usar las TIC en clase entusiasma a los estudiantes con discapacidades

19. Los estudiantes están más familiarizados con las TIC que yo

20. El uso de las TIC aumenta el interés propio de los estudiantes con discapacidad en un entorno inclusivo

21. El uso de las TIC en el entorno escolar general mejora la autoestima de los estudiantes con discapacidad

22. En la escuela, existe cooperación entre los docentes para implementar escenarios de enseñanza utilizando las TIC

23. Las TIC brindan la oportunidad de colaboración entre docentes de diferentes especialidades $\mathrm{e}$ incluso de distintos entornos escolares (contexto de educación general e inclusivo).

24. El Consejo de Educación Especial de la Escuela alienta a los docentes a utilizar el uso de las TIC en el contexto de los programas de educación inclusiva

25. Las TIC del centro me permiten evaluar sistemáticamente mis resultados de enseñanza y aprendizaje

26. Tengo la infraestructura esencial en mi escuela para aplicar las TIC a mi clase en el contexto de los programas de educación inclusiva

27. El director, en la escuela donde trabajo, alienta a los maestros a participar en el uso de las TIC en el contexto de los programas de educación inclusiva

28. El plan de estudios facilita la implementación de las TIC

29. La educación inclusiva tiene un efecto positivo en el desarrollo social y emocional de los estudiantes con discapacidad

30. El uso de las TIC funciona como una ayuda didáctica para la aplicación de programas inclusivos

31. Las necesidades de los estudiantes con discapacidades pueden satisfacerse mejor en un entorno especial y separado

32. Las TIC promueven el trabajo en equipo de los estudiantes en entornos de integración

Fuente: Elaboración propia.

Tratando de comprobar la fiabilidad de las dimensiones (factores) arrojados por el AFE, se ha procedido a comprobar el valor de Alpha e igualmente, se denominó a los mismos, siendo como vemos en la Tabla 3, un valor optimo o alto (MATEO, 2012) para 
cada una de las mismas.

Tabla 3: Valor de Alpha de las dimensiones.

\begin{tabular}{|l|c|}
\hline \multicolumn{1}{|c|}{ Dimensiones } & Valor de Alpha \\
\hline Auto percepción & .831 \\
\hline TIC+educación inclusiva+alumnado & .721 \\
\hline Visiones del centro & .866 \\
\hline Desarrollo de la formación & .816 \\
\hline
\end{tabular}

Fuente: Elaboración propia.

\section{Resultados}

\subsection{Estudio descriptivo}

Como se puede consultar en la Tabla 4, el profesorado de educación primaria y secundaria griego participante en el estudio, considera ser cauteloso y seguro en el empleo de las TIC en el ámbito educativo, dado que no cree que limiten las interacciones sociales. Igualmente, tampoco piensan que el no conocerlas haga que no las utilicen pues les gusta emplearlas, aunque no necesitan probar nuevas herramientas digitales para su práctica docente y tampoco considera que sus estudiantes estén más formados que ellos en su utilización educativa. A ello se ha de unir que contemplen que el plan de estudios no facilita la implementación de las TIC en el proceso formativo.

Por otra parte, opinan que, si tuvieran los recursos, tales como ordenadores, en sus aulas las emplearían más, dado que los ven como un recurso útil en el desarrollo de los programas de educación inclusiva, a la vez que se muestran muy interesados en aquellos que les ayuden a mejorar esta, pues piensan que incluyen actividades inclusivas y que en consecuencia consideran que las TIC mejorarían la calidad del proceso de enseñanza. Junto a todo ello se sienten optimistas, dado que ven que los recursos digitales les permitiría trabajar con otros colegas tanto de especialidad como del centro o de otras instituciones educativas o niveles.

Con respecto a los estudiantes, se expresan positivos en opinar que las TIC en general y en concreto los ordenadores, ayudan a los estudiantes en su proceso de aprendizaje (DA SILVA; DE CARVALHO; RAMOS, 2020). En concreto los alumnos que presentan discapacidades cognitivas o intelectuales pueden mejorar su rendimiento, así como su socialización y despertar en ellos un mayor interés por su formación dado que se puede potenciar el trabajo en equipo.

Por último, con respecto a la institución educativa, contemplan que desde la dirección de sus centros se está apoyando la incorporación de las TIC a las dinámicas inclusivas del aula, y que el sistema educativo permite que el desarrollo social y emocional de los alumnos se pueda realizar. También ponen de relieve que la propia institución puede satisfacer las necesidades de los alumnos discapacitados cognitiva e intelectualmente y que promueve el trabajo en equipo entre ellos. 
Tabla 4: Valores estudio descriptivo.

\begin{tabular}{|c|c|c|c|c|c|c|c|c|c|c|c|c|}
\hline & \multicolumn{2}{|c|}{1} & \multicolumn{2}{|c|}{2} & \multicolumn{2}{|c|}{3} & \multicolumn{2}{|c|}{4} & \multicolumn{2}{|c|}{5} & \multirow[b]{2}{*}{ M. } & \multirow[b]{2}{*}{ D.T. } \\
\hline & $\mathrm{F}$. & $\%$ & F. & $\%$ & F. & $\%$ & F. & $\%$ & F. & $\%$ & & \\
\hline Ítem 1 & 121 & 32.1 & 208 & 55.2 & 22 & 5.8 & 21 & 5.6 & 5 & 1.3 & 1.89 & .843 \\
\hline Ítem 2 & 136 & 36.1 & 190 & 50.4 & 10 & 2.7 & 41 & 10.9 & & & 1.88 & .900 \\
\hline Ítem 3 & & & 6 & 1.6 & 7 & 1.9 & 113 & 30 & 251 & 66.6 & 4.62 & .609 \\
\hline Ítem 4 & & & & & 21 & 5.6 & 148 & 39.3 & 208 & 55.2 & 4.50 & .602 \\
\hline Ítem 5 & & & & & 11 & 2.9 & 251 & 66.6 & & & 4.28 & .509 \\
\hline Ítem 6 & & & & & & & 176 & 46.7 & 201 & 53.3 & 4.53 & .500 \\
\hline Ítem 7 & & & & & & & 124 & 32.9 & 253 & 67.1 & 4.67 & .470 \\
\hline Ítem 8 & 233 & 61.8 & 122 & 32.4 & 5 & 1.3 & 14 & 4.5 & & & 1.49 & .740 \\
\hline Ítem 9 & 36 & 9.5 & 161 & 42.7 & 31 & 8.2 & 124 & 32.9 & & & 2.84 & 1.176 \\
\hline Ítem 10 & & & & & 2 & .5 & 223 & 59.2 & 152 & 40.3 & 4.40 & .501 \\
\hline Ítem 11 & 70 & 18.6 & 246 & 65.3 & 37 & 9.8 & 24 & 6.4 & & & 2.04 & .734 \\
\hline Ítem 12 & 25 & 6.6 & 34 & 9 & 54 & 14.3 & 201 & 53.3 & 63 & 16.7 & 3.64 & 1.070 \\
\hline Ítem 13 & & & 38 & 10.1 & 53 & 14.1 & 162 & 43 & 124 & 32.9 & 3.99 & .935 \\
\hline Ítem 14 & & & 10 & 2.7 & 1 & .3 & 220 & 58.4 & 146 & 38.7 & 4.33 & .622 \\
\hline Ítem 15 & & & 5 & 1.3 & 62 & 16.4 & 171 & 45.4 & 139 & 36.9 & 4.18 & .746 \\
\hline Ítem 16 & & & & & 8 & 2.1 & 269 & 71.4 & 100 & 26.5 & 4.24 & .477 \\
\hline Ítem 17 & & & 7 & 1.9 & 11 & 2.9 & 195 & 51.7 & 164 & 43.5 & 4.37 & .635 \\
\hline Ítem 18 & & & 11 & 2.9 & 13 & 3.4 & 195 & 51.7 & 158 & 41.9 & 4.33 & .682 \\
\hline Ítem 19 & 154 & 40.8 & 194 & 51.5 & 18 & 4.8 & 11 & 2.9 & & & 1.70 & .695 \\
\hline Ítem 20 & & & 81 & 2.1 & 19 & 5 & 249 & 66 & 101 & 26.8 & 4.18 & .611 \\
\hline Ítem 21 & & & 6 & 1.6 & 15 & 4 & 229 & 60.7 & 127 & 33.7 & 4.27 & .609 \\
\hline Ítem 22 & & & 121 & 32.1 & 83 & 22 & 134 & 35.5 & 39 & 103 & 3.24 & 1.017 \\
\hline Ítem 23 & & & & & 1 & .3 & 174 & 46.2 & 202 & 53.6 & 4.53 & .505 \\
\hline Ítem 24 & 7 & 1.9 & 54 & 14.3 & 195 & 51.7 & 91 & 24.1 & 30 & 8 & 3.22 & .855 \\
\hline Ítem 25 & & & & & 9 & 2.4 & 207 & 54.9 & 161 & 42.7 & 4.40 & .538 \\
\hline Ítem 26 & 45 & 11.9 & 139 & 36.9 & 30 & 8 & 121 & 32.1 & 42 & 11.1 & 2.94 & 1.270 \\
\hline Ítem 27 & & & 80 & 21.2 & 79 & 21 & 184 & 48.8 & 34 & 9 & 3.46 & .925 \\
\hline Ítem 28 & 41 & 10.9 & 117 & 31 & 81 & 21.5 & 102 & 27.1 & 36 & 9.5 & 2.93 & 1.182 \\
\hline Ítem 29 & & & & & 12 & 3.2 & 207 & 54.9 & 158 & 41.9 & 4.39 & .549 \\
\hline Ítem 30 & & & & & 6 & 1.6 & 271 & 71.9 & 100 & 26.5 & 4.25 & .469 \\
\hline Ítem 31 & 47 & 12.5 & 85 & 22.5 & 49 & 13 & 124 & 32.9 & 72 & 19.1 & 3.24 & 1.329 \\
\hline Ítem 32 & & & & & 31 & 8.29 & 233 & 61.8 & 1.3 & 30 & 4.22 & .579 \\
\hline
\end{tabular}

Fuente: Elaboración propia.

\subsection{Estudio inferencial}

Para determinar la existencia o no de diferencias en la muestra atendiendo al género, se ha podido constatar que estas no se producen, tras realizar la prueba $\mathrm{T}$ de Student. En la misma línea para comprobar que hubiera diferencias en función de la edad, en línea con los datos de la prueba anterior, se ha procedió a aplicar una ANOVA, la cual ha arrojado, también la no existencia de diferencias estadísticamente significativas en función de esa variable.

\subsection{Estudio correlacional}

Atendiendo a las 4 dimensiones establecidas en el AFE realizado anteriormente, se 
ha procedido a la realización de un estudio correlacional de las mismas.

* Dimensión 1. Auto percepción: En lo que se refiere a los ítems de la dimensión 1, comprobamos que no hay una correlación significativa a nivel ni de .05 ni .01, salvo entre el ítem 6 (El uso de nuevas herramientas digitales en programas de enseñanza inclusiva me interesa) hacia el 10 (Las computadoras ayudan a los estudiantes con diferentes estilos de aprendizaje a aprender mejor y participar más), [R=-.127(*); $\mathrm{P}=.013]$ y del 3 (Si tuviera una computadora en mi clase, la usaría en el proceso de enseñanza) hacia el 2 (Evito usar las TIC porque no tengo el conocimiento necesario) [R=-.102(*); $\mathrm{P}=.049$ ] y hacia el 5 (EI uso de las TIC en el aula de hoy incluye actividades inclusivas) $\left[R=-.125\left(^{*}\right) ; P=.015\right]$, cuya correlación es a nivel de confianza de 05.

* Dimensión 2. TIC+ educación inclusiva+alumnado: Entre los ítems que conforman esta dimensión, solo encontramos correlaciones entre el 14 (Los estudiantes con discapacidad cognitiva e intelectual pueden obtener una mejora académica debido a la educación inclusiva y a la implementación de las TIC en el aula) y el 12 (La inclusión suena bien en teoría pero no funciona bien en la práctica) $\left[\mathrm{R}=.110\left(^{*}\right) ; \mathrm{P}=.033\right]$; y entre el 19 (Los estudiantes están más familiarizados con las TIC que yo) con el 15 (El uso frecuente de las TIC contribuye a la socialización de los estudiantes con discapacidad cognitiva e intelectual) [R=-.109(*); $\mathrm{P}=.034]$, y el 17 (Los escenarios de enseñanza con TIC atraen el interés de los estudiantes con discapacidad cognitiva e intelectual) $\left[\mathrm{R}=-.108\left(^{\star}\right) ; \mathrm{P}=.036\right]$ produciéndose estas, al igual que en la dimensión anterior, a un nivel de significación de .05. Hay que destacar que en el caso del ítem 16 (La implementación de las TIC puede mejorar el nivel de aprendizaje de los estudiantes con discapacidades cognitivas e intelectuales) hay una correlación significativa con el 20 (El uso de las TIC aumenta el interés propio de los estudiantes con discapacidad cognitiva e intelectual en un entorno inclusivo) $\left[\mathrm{R}=, 163{ }^{(* *)}\right.$; $\left.\mathrm{P}=.001\right]$ a un nivel de confianza de .01.

* Dimensión 3. Visiones del centro: En esta dimensión, solo encontramos una correlación significativa a nivel de confianza de $\mathrm{n}=.05$, estando ésta entre el ítem 27 ( $E l$ director, en la escuela donde trabajo, alienta a los maestros a participar en el uso de las TIC en el contexto de los programas de educación inclusiva) y el 22 (En la escuela, existe cooperación entre los docentes para implementar escenarios de enseñanza utilizando las TIC) $\left[\mathrm{R}=, 120\left(^{*}\right) ; \mathrm{P}=.020\right]$.

* Dimensión 4. Desarrollo de la formación: En la cuarta dimensión se ha constatado que no hay correlación significativa entre los ítems que la conforman.

\section{Discusión y conclusiones}

Como señala Echeita (2017) la educación inclusiva no es una aspiración, creemos que debe ser una realidad, es "un proceso por el que cualquier persona participa de forma activa en su aprendizaje, progresando y accediendo en equidad al mismo" (SAMPEDRO; MALDONADO, 2017, p. 90). Esto junto con las grandes posibilidades que ofrecen las TIC a la enseñanza y el aprendizaje hacen que el escenario inclusivo digital sea una realidad. Como ya hemos señalado antes un elemento cardinal en este escenario es la figura del docente. Esto, sus creencias, visiones y experiencias harán que las TIC tengan o no una presencia en la educación inclusiva (FERNÁNDEZ BATANERO; REYES REBOLLO; EL 
HOMRANI, 2018; MARTÍNEZ; GUTIÉRREZ; FERNÁNDEZ, 2018).

Los resultados alcanzados nos indican que no hay diferencias en torno al género y al posicionamiento del profesorado griego frente al uso de las TIC en las aulas inclusivas, al igual que sucede en el trabajo de Marín con docentes españoles (2017). Con respecto a la edad y las metodologías docentes, vemos que tampoco se producen diferencias, por lo que podemos inferir que el profesorado griego que trabaja en centros o espacios inclusivos, independientemente de la edad, considera que es un elemento que debe ser incorporado a su aula, como refleja los trabajos de Fernández, Román y El Homrani (2017), Silva y Rodríguez (2018). Atendiendo a los años de experiencia profesional como docentes en el ámbito de la educación inclusiva, tampoco se han alcanzado diferencias estadísticamente significativas, como sucede en otros trabajos (MARÍN, 2013).

Atendiendo a las dimensiones que el AFE ha señalado comprobamos que el profesorado griego al igual que en otros contextos como el colombiano, mexicano, congoleño o español (FERNÁNDEZ et al., 2017; LAITON et al., 2017; MAESTRE; NAIL; RODRÍGUEZ, 2017; PEGALAJAR, 2017; SAMPEDRO; MALDONADO, 2017; SILVA; RODRÍGUEZ, 2018; CISTERNAS; LOBOS, 2019; WAFUNGA; RODRÍGUEZ-GARCÍA; SOLA, 2019), no tiene una visión de sí mismo positiva en lo que se refiere a su formación para el empleo de las TIC en sus aulas, pero al igual que en otros escenarios o niveles formativos consideran interesante y que se encuentran abiertos a trabajar en escenarios de cooperación y colaboración entre profesores, en este caso a diferencia de los hallazgos de Laiton et al. (2017).

Con respecto a la visión que tienen del alumnado inclusivo, piensan que estos deben incorporarse a las aulas ordinarias (regulares) (LAITON et al., 2017), y que ello junto con el empleo de las TIC en su proceso formativo beneficiará su proceso de aprendizaje, al igual que las investigaciones de Vega-Moreno, Cufí, Rueda y Llinás (2016), Cámara et al., (2017), Fernández del Río y Barreira (2017), Marín-Díaz (2017) y Chaparro, Escalante y Samacá (2019). Es significativo que opinen que los estudiantes inclusivos incluso están preparados mejor que ellos para la utilización de los recursos tecnológicos en los procesos de capacitación, por lo que podemos concluir en este aspecto que su visión del alumno inclusivo digital, es muy alto.

Poniendo el acento en la institución educativa griega en general, los profesores griegos al igual sucede en otros contextos (FERNÁNDEZ BATANERO; ROMÁN GRAVÁN; EL HOMRAN, 2017; FERNÁNDEZ BATANERO; REYES REBOLLO; EL HOMRANI, 2018; VEGA-MORENO et al., 2016), piensan que están arropados en este caso por su equipo directivo y la Comisión de Educación Especial y a diferencia de los datos aportados por Hamburg y Bucksch (2017). Igualmente comparten con los datos alcanzados por Marín, Vidal, Peirats y San Martín (2019), que disponen de los recursos para su implementación y promover la colaboración entre el profesorado de manera individual y a través de equipos de trabajo colaborativo a diferencia de los aportados por la investigación de Yusof, Daniel, Low y Aziz (2014) y de Laiton et al. (2017), quienes consideraban que no se daban las condiciones para que ello se llevara a buen puerto.

La última dimensión marcada por el AFE, referida al desarrollo de la formación, desde una perspectiva general, los docentes griegos opinan que el plan de estudios se puede abordar desde una perspectiva inclusiva digital (CORTÉS; GONZÁLEZ; SÁNCHEZ, 2018; SILVA; RODRÍGUEZ, 2018; VEGA-MORENO et al., 2016), así como el promover programas de formación entre y para docentes así como para alumnos (GARCÍA MIRANDA, 2016; PEGALAJAR, 2017; ÁVALOS et al., 2018; MARTÍNEZ; 
GUTIÉRREZ; FERNÁNDEZ, 2018; SILVA; RODRÍGUEZ, 2018).

En definitiva, el estudio realizado entre profesorado griego de Educción Primaria y Secundaria ha puesto de relieve que, para ellos, es clave el uso de las TIC para el desarrollo de una educación inclusiva y que ello está íntimamente vinculado el empleo en concreto de ordenadores no solo por parte de ellos sino también de su alumnado, dado que ello permitirá a estos participar de manera activa en las acciones formativas, así como mejorar su proceso de aprendizaje. Por otra parte, es significativo que al igual que sucede en otros contextos geográficos la escasa formación digital y una visión negativa de sí mismo con respecto a su competencia digital que manifiesta tener, se reclamen acciones formativas directas en formatos dinámicos e innovadores que ayuden a superar esta circunstancia.

Por último, debemos promover una educación inclusiva soportada en recursos digitales que haga que pensamientos negativos - como el considerar que la educación inclusiva en teoría es plausible pero que en la práctica no lo es -, pues las acciones de innovación docente donde se vincula el uso de las TIC y la educación inclusiva llevadas a cabo por Fernández (2013), Hamburg y Bucksch (2017), García Miranda (2016), Cámara et al. (2017) o Arnaiz, de Haro y Azorín (2018) o de investigación como Da silva, de Carvalho y Ramos (2020) o Chaves (2017), por citar algunas, pone de relieve que ello es posible lograr.

\section{Referencias}

ARNAIZ, P., DE HARO, R.; AZORÍN, C. M. Redes de apoyo y colaboración para la mejora de la educación inclusiva. Profesorado, Revista de Currículum y Formación del Profesorado, v. 22, n. 2, p. 29-49, 2018. Disponible en: https://revistaseug.ugr.es/index.php/profesorado/article/view/7713. Acceso en: 23 abr. de 2019.

ÁVALOS, C.; REDONDO, V.; ROJAS, D.; SALAZAR, J. Metodologías inclusivas de aprendizaje mediante el uso de TIC: opiniones de estudiantes y docentes costarricenses. Revista Postgrado y Sociedad, v. 16, n. 1, p. 53-69, 2018. doi: https://doi.org/10.22458/rpys.v16i1.2098. Acceso en: 23 abr. 2019.

CABERO, J.; VALENCIA, R. TIC para la inclusión: una mirada desde Latinoamérica. Aula Abierta, v. 48, n. 2, p. 139-146, 2019. doi: https://doi.org/10.17811/rifie.48.2.2019.139-146. Acceso en: 12 abr. 2020.

CÁMARA, A.; DÍAZ, E.; ORTEGA, J. M. Aprendizaje-servicio en la universidad: ayudando a la escuela a atender a la diversidad a través de las TIC. Bordón, v. 69, n. 3, 1-16, 2017. doi: https://doi.org/10.13042/Bordon.2017.51320. Acceso en: 23 abr. 2019.

CHAPARRO, C.; ESCALANTE, G.; SAMACÁ, E. Las TIC como estrategia didáctica dentro del proceso educativo de estudiantes universitarios sordos. Cuidado y Ocupación Humana, $\quad$ v. $2019 . \quad 2$ Disponible 2 en: http://revistas.unipamplona.edu.co/ojs_viceinves/index.php/COH/article/view/3393. Acceso en: 3 nov. 2019. 
CHAVES, F. Análise da produção da escrita e do discurso do surdo no Facebook. Texto Livre: Linguagem e Tecnologia, Belo Horizonte, v. 10, n. 1, p. 53-65, 2017. doi: 10.17851/1983-3652.10.1.53-65. Acceso en: 28 jun. 2020.

CISTERNAS, T.; LOBOS, A. Profesores nóveles de enseñanza básica: Dilemas, estrategias y obstáculos para abordar los desafíos de una educación inclusiva. Revista Latinoamericana de Educación Inclusiva, v. 13, n. 1, p. 37-53, 2019. doi: http://dx.doi.org/10.4067/S0718-73782019000100037. Acceso en: 3 abr. 2020.

CORTÉS, P.; GONZÁLEZ, B.; SÁNCHEZ, M. F. Agrupamientos escolares y retos para la educación inclusiva en infantil y primaria, Tendencias Pedagógicas, v. 32, p. 75-90, 2018. doi: 10.15366/tp2018.32.006. Acceso en: 3 nov. 2019.

CUMMING J.; TONES M., DAY C.; HECK, E. Enhancing Inclusive Education Through Teacher Education Reforms. En: WYATT-SMITH C.; ADIE L. (eds.). Innovation and Accountability in Teacher Education. Teacher Education, Learning Innovation and Accountability Springer, Singapore, 2018, p. 201-220. doi: https://doi.org/10.1007/978981-13-2026-2_13. Acceso en: 3 nov. 2019.

DA SILVA, J. A; DE CARVALHO, M. E.; RAMOS R. V. As tecnologias digitais da informação e comunicação como mediadoras na alfabetização de pessoas com transtorno do espectro do autismo: uma revisão sistemática da literatura, Texto Livre: Linguagem e Tecnologia, Belo Horizonte, v. 13, n. 1, p. 45-64, 2020. doi: 10.17851/1983-3652.13.1.4564. Acceso en: 28 jun. 2020.

ECHEITA, G. Educación inclusiva. Sonrisas y lágrimas. Aula Abierta, v. 46, n. 2, p. 17-24, 2017. doi: https://doi.org/10.17811/rifie.46.2017.17-24. Acceso en: 3 nov. 2019.

FERNÁNDEZ BATANERO, J. M.; REYES REBOLLO, M. M.; EL HOMRANI, M. TIC y discapacidad. Principales barreras para la formación del profesorado. EDMETIC, v. 7, $\mathrm{n}$. 1, p. 1-25, 2018. https://doi.org/10.21071/edmetic.v7i1.9656

FERNÁNDEZ BATANERO, J. M.; ROMÁN GRAVÁN, P.; EL HOMRAN, M. TIC y discapacidad. Conocimiento del profesorado de educación primaria em Andalucía. Aula Abierta, v. 46, p. 65-72, 2017. https://doi.org/10.17811/

FERNÁNDEZ DEL RÍO, A. B.; BARREIRA, A. J. El cortometraje como herramienta innovadora para el alumnado con Altas Capacidades en Educación Primaria. Innoeduca. International Journal of Technology and Educational Innovation, v. 3, n. 1, p. 28-36, 2017. doi: http://dx.doi.org/10.24310/innoeduca.2017.v3i1.1962. Acceso en: 3 nov. 2019.

FERNÁNDEZ, J. M. Competencias docentes y educación inclusiva. Revista Electrónica de Investigación Educativa, v. 15, n. 2, p. 82-99, 2013. Recuperado de: https://redie.uabc.mx/redie/article/view/445/610. Acceso en: 3 nov. 2019.

FIGUEREDO, V. TIC y educación inclusiva en Europa. En: PADILLA, D.; LÓPEZ, R.; AGUILAR, J. M. (eds.). Psicología, salud y educación. Almería: Editorial Universidad de 
Almería, 2018, p. 391-397.

FUENTE-COBO, C. Públicos vulnerables y empoderamiento digital: el reto de una sociedad e-inclusiva. El profesional de la información, v. 26, n. 1, p. 5-12, 2017. doi: https://doi.org/10.3145/epi.2017.ene.01

GARCÍA, M. G. Las TIC en la educación inclusiva. incorporación de las AULAS VIRTUALES como estrategia para la Enseñanza y Aprendizaje de CIENCIAS en ProCES Semipresencial. Revista Temas de Profesionalización Docente, v. 2, p. 77-83, 2019. Disponible en: http://ojs.cfe.edu.uy/index.php/rev_temas/article/view/368/217 Acceso en: 3 nov. 2019.

GARCÍA MIRANDA, D. Material didático digital: nova forma de o aluno surdo "ler" e "interagir" com os conteúdos educacionais? Texto Livre: Linguagem e Tecnologia, Belo Horizonte, v. 9, n. 2, p. 185-198, 2016. doi: 10.17851/1983-3652.9.2.185-198. Acceso en: 28 jun. 2020.

HAMBURG, I.; BUCKSCH, S. Inclusive education and digital social innovation. Advances in Social Sciences Research Journal, v. 4, n. 5, p. 162-169, 2017. doi: https://doi.org/10.14738/assrj.45.2861. Acceso en: 3 nov. 2019.

HERNÁNDEZ, R.; FERNÁNDEZ, C.; BAPTISTA, P. Metodología de la investigación. (4ª . ed.). México: McGraw Hill Interamericana, 2006.

KORKOUTAS, E. Reflexiones sobre la inclusión y los modelos de intervención en la escuela para el alumnado con necesidades educativas especiales. Campo Abierto, v. 30, n. $2, \quad$ p. $128-138, \quad 2011 . \quad$ Disponible http://dehesa.unex.es/bitstream/handle/10662/5520/0213-9529_30_127.pdf? sequence=1\&isAllowed=y. Acceso en: 14 mar. 2020.

LAITON, E. V.; GÓMEZ, S. E.; SARMIENTO, R. E.; MEJÍA, C. Competencia de Prácticas Inclusivas: Las TIC y la Educación inclusiva en el desarrollo profesional docente. Sophia, v. 13, p. 2, p. 82-95, 2017. doi: https://doi.org/10.18634/sophiaj.13v.2i.502. Acceso en: 14 mar. 2020.

LEIVA, J. J.; GÓMEZ, M. A. La educación inclusiva como constructo pedagógico en el alumnado universitario de educación primaria. Revista Educación Inclusiva, v. 8, n. 2, p. 185-200, 2015.2 Disponible en: https://www.revistaeducacioninclusiva.es/index.php/REl/article/view/114. Acceso en: 14 mar. 2020.

LEY 4488/2017. Greece. Pension and other insurance provisions, strengthening worker protection, rights of persons with disabilities and other provisions. Athens: National Printing House. https://www2.deloitte.com/content/dam/Deloitte/gr/Documents/tax/gr_tax_alert_recent_cha nges_labour_soc_security_Law_en_noexp.pdf. Acceso en: 10 mar. 2020.

MAESTRE, M. M.; NAIL, O.; RODRÍGUEZ, A. J. Desarrollo de competencias TIC y para la 
educación inclusiva en la formación inicial práctica del Profesorado. Bordón, v. 69, n. 3, p. 57-72, 2017. doi: 10.13042/Bordon.2017. 51110. Acceso en: 10 de marzo de 2020.

MANTILLA, F. Métodos Estadísticos Exploratorios y Confirmatorios para Análisis de Datos. Ecuador: ESPE, 2015.

MARÍN, D.; VIDAL, M.; PEIRATS, J.; SAN MARTÍN, Á. Competencia digital transversal en la formación del profesorado, análisis de una experiencia. Innoeduca. International Journal of Technology and Educational Innovation, v. 5, n. 1, p. 4-12, 2019. doi: http://dx.doi.org/10.24310/innoeduca.2019.v5i1.4890. Acceso en: 10 mar. 2020.

MARÍN, V. TIC's para la educación inclusiva. Vigo: Da Vinci, 2013.

MARÍN, V. Las TIC inclusivas o la inclusividad de las TIC. EDMETIC, Revista de Educación Mediática y TIC, v. 7, n. 1, p. I-III, 2018 . doi: https://doi.org/10.21071/edmetic.v7i1.10515. Acceso en: 10 mar. 2020.

MARÍN-DÍAZ, V. The relationships between Augmented Reality and inclusive education in Higher Education. Bordón, v. 69, n. 3, p. 125-142, 2017. doi: http://dx.doi.org/10.13042/Bordon.2017.51123. Acceso en: 10 mar. 2020.

MARÍN-DÍAZ, V. ICT-Based Inclusive Education. En: TATNALL, A. (eds.), Encyclopedia of Education and Information Technologies. Springer, Cham, 2020, p. 1-18. doi: https:// doi.org/10.1007/978-3-319-60013-0.

MARTÍNEZ, S.; GUTIÉRREZ, J. J.; FERNÁNDEZ, B. Percepciones y uso de las TIC en las aulas inclusivas. Un estudio de caso. EDMETIC, Revista de Educación Mediática, v. 7, n. 1, p. 87-106, 2018. doi: https://doi.org/10.21071/edmetic.v7i1.10132. Acceso en: 10 mar. 2020.

MATEO, J. La investigación ex post - facto. En: BISQUERRA, R. (coord.). Metodología de la investigación educativa (3a. ed.). Madrid: La Muralla, 2012, p. 195-229.

PADELIADU, S.; PAPANIKOLAOU, G.; GIAZITZIDOU, S. Implementing inclusive education in Greece through co-teaching. Conference: 8th International Scientific Conference for Special Education and Rehabilitation. At: Belgrade, Serbia, 2014. Disponible https://www.researchgate.net/publication/274380022 Implementing inclusive education i n_Greece through_co-teaching. Acceso en: 10 mar. 2020.

PAPAGEORGIOU, M. Critical Approaches to Inclusive Education. Universal Review, v.VIII, n. I, p. I, 2019. doi: http://dx.doi.org/10.239/ssrn.3339212. Acceso en: 10 mar. 2020.

PARRILLA, A. Acerca del origen y sentido de la educación inclusiva. Revista de Educación, $\quad$ v. 327, p. 11-29, 2002. Disponible en; http://www.educacionyfp.gob.es/dam/jcr:1c6b8d39-de6f-429a-9db2-67b52a1a6e63/re327pdf.pdf. Acceso en: 10 mar. 2020. 
PASSEY, D. Developing inclusive practices with technologies for online teaching and learning: a theoretical perspective. Bordón, v. 69, n. 3, p. 25-40, 2017. doi: https://doi.org/10.13042/Bordon.2017.53523. Acceso en: 10 mar. 2020.

PEGALAJAR, M. C. Educación inclusiva y TIC: análisis de actitudes en futuros docentes. JETT, Journal for Educators, Teachers and Trainers, v. 6, n. 1, p. 250-264, 2015. Disponible en: http://jett.labosfor.com/index.php/jett/article/view/182/222. Acceso en: 10 mar. 2020.

PEGALAJAR, M. C. El futuro docente ante el uso de las TIC para la educación inclusiva. Digital Education Review, v. 31, p. 131-148, 2017. Disponible en: http://revistes.ub.edu/index.php/der/article/view/16016/pdf. Acceso en: 10 mar. 2020.

RICO, M. J.; BOSAGAIN, X. Pensamiento computacional: rompiendo brechas digitales y educativas. EDMETIC, Revista de Educación Mediática y TIC, v. 7, n. 1, p. 26-42, 2018, doi: https://doi.org/10.21071/edmetic.v7i1.10039. Acceso en: 10 mar. 2020.

ROMERO, S. J.; GONZÁLEZ, I.; GARCIA, A.; LOZANO, A. Herramientas tecnológicas para la educación inclusiva. Tecnología, Ciencia y Educación, n. 9, p. 83-112, 2018. Disponible en: https://tecnologia-ciencia-edu. Acceso en: 3 abr. 2020.

SALTA, K.; KOULOUGLIOTIS, D. Formación de Profesores de Química: Experiencia Internacional y el Caso Griego, 2014. Disponible en: https://chemistrynetwork.pixelonline.org/files/TET_papers/EL/EL_Teacher\%20Training_ES.pdf. Acceso en: 10 mar. 2020.

SAMPEDRO, B. E.; MALDONADO, G. A. Valoraciones de los estudiantes mexicanos y españoles sobre el uso de las TIC como recurso para trabajar la educación inclusiva. Bordón, v. 69, n. 3, p. 89-106, 2017. doi:10.13042/Bordon.2017.51237. Acceso en: 10 mar. 2020.

SILVA, G.; RODRÍGUEZ, F. P. Una mirada hacia las TIC en la educación de las personas con discapacidad y con trastorno del espectro autista: análisis temático y bibliográfico. EDMETIC, Revista de Educación Mediática y TIC, v. 7, n. 1, p. 43-65, 2018. https://doi.org/10.21071/edmet. Acceso en: 3 abr. 2020.

THOMAS, J.; BARRAKET, J.; EWING, S.; MACDONALD, T.; MUNDELL, M.; TUCKER, J. Measuring Australia's Digital Divide: The Australian Digital Inclusion Index 2016. Melbourne: Swinburne University of Technology, 2016. doi: www.dx.doi.org/10.4225/50/57A7D17127384.

TOLEDO, P. Las tecnologías de la información, la comunicación y la inclusión educativa. En: BARROSO, J.; CABERO, J. (eds.). Nuevos escenarios digitales. Las tecnologías de la información y la comunicación aplicadas a la formación y el aprendizaje curricular. Madrid: Pirámide, 2013, p. 411-426.

UNESCO. Guía para asegurar la inclusión y la equidad en la educación. París: UNESCO, 2017. 
VEGA-MORENO, D.; CUFÍ, X.; RUEDA, M. J.; LLINÁS, D. Integración de robótica educativa de bajo coste en el ámbito de la educación secundaria para fomentar el aprendizaje por proyectos. IJERI: International Journal of Educational Research and Innovation, $\quad$ v. $6, \quad$ p. $162-175, \quad 2016 . \quad$ Disponible en: https://www.upo.es/revistas/index.php/IJERI/article/view/1653. Acceso en: 10 mar. 2020.

WAFUNGA, H.; RODRÍGUEZ-GARCÍA, A.; SOLA, J. Competencias profesionales de los profesores de Biología de las escuelas de primer ciclo de Enseñanza Secundaria en Benguela (Angola). Innoeduca. International Journal of Technology and Educational Innovation, v. 5, n. 2, p. 114-121, 2019. doi: http://dx.doi.org/10.24310/innoeduca.2019.v5i2.260. Acceso en: 10 mar. 2020.

YUSOF, A. M.; DANIEL, E. G. S.; LOW, W. Y.; AZIZ, K. A. Teachers' perception of mobile edutainment for special needs learners: the Malaysian case. International Journal of Inclusive Education, v. 18, n. 12, p. 1237-1246, 2014. doi: 10.1080/13603116.2014.885595. Acceso en: 10 mar. 2020.

ZAPPATA, D.; KÖPPEL, A.; SUCHODOLISKI, M. Inclusión de TIC en escuelas para alumnos con discapacidad visual. Buenos Aires: Ministerio de Educación de la Nación, 2011. 